\title{
Inertia-Induced Breakdown of Acoustic Sorting Efficiency at High Flow Rates
}

\author{
Eva Undvallø, ${ }^{1}$ Fabio Garofalo, ${ }^{2}$ Giuseppe Procopio, ${ }^{2}$ Wei Qiu $\odot,{ }^{1}$ Andreas Lenshof, ${ }^{1}$ \\ Thomas Laurell, ${ }^{1}$ and Thierry Baasch $\circledast^{1, *}$ \\ ${ }^{1}$ Departement of Biomedical Engineering, Lund University, Sweden \\ ${ }^{2}$ Dipartimento di Ingegneria Chimica Materiali e Ambiente, Universitá di Roma La Sapienza, Italy
}

(Received 17 November 2021; revised 12 January 2022; accepted 19 January 2022; published 4 March 2022)

\begin{abstract}
The clinical utility of microfluidic techniques is often hampered by an unsatisfying sample throughput. Here, the effect of inertial forces on acoustofluidic particle sorting at high sample throughputs is investigated experimentally and theoretically. Polystyrene particles are acoustically prefocused to obtain precise trajectories. At increased flow rates it is observed that the particle stream is displaced towards the channel center, and above specific flow settings the particles spill over into the center outlet. This effect, coined the spillover effect, illustrates the complex interplay of viscous and inertial forces inside the microchannel. The effect is due to increased bending of the separatrices at the inlet and outlets and not due to the wall-lift force. The impact of the spillover effect on the separation of two different-sized particles is subsequently studied. Efficient sorting is done for subcritical splitting ratios and flow rates, but for close to critical settings or beyond, there is a breakdown of the acoustofluidic separation.
\end{abstract}

DOI: 10.1103/PhysRevApplied.17.034014

\section{INTRODUCTION}

Acoustophoretic particle manipulation in microfluidic systems, i.e., the active control of particles or cells by the means of acoustic waves, has been used to separate lipids from blood [1], rapidly control raw-milk quality [2], enrich tumor cells from blood samples [3], further reduce remaining white-blood-cell background through negative selection [4] and separate leukocyte subpopulations [5] amongst others. As with any microfluidic method, the sample throughput is key to a successful application. If rare cells, such as circulating tumor cells (CTCs) or CTC clusters, are to be isolated from a sample size of several milliliters, a whole-blood throughput in the hundreds of microliters per minute or higher should be achieved for the application to be relevant in the clinical setting.

Most commonly, whole-blood samples are diluted before processing through a microfluidic device, and therefore it is useful to distinguish between the flow rate in the channel and the whole-blood throughput. Typical values for the per-channel whole-blood throughput in microfluidic applications are in the range of a few microliters per minute, and can reach more than $100 \mu \mathrm{lmin}^{-1}$

\footnotetext{
*Thierry.Baasch@bme.lth.se

Published by the American Physical Society under the terms of the Creative Commons Attribution 4.0 International license. Further distribution of this work must maintain attribution to the author(s) and the published article's title, journal citation, and DOI. Funded by Bibsam.
}

for high-throughput applications. For example, the whole blood throughput for inertia-based separation of white blood cells from whole-blood ranges from a whole-blood throughput of $1.5 \mu 1 \mathrm{~min}^{-1}$ [6] over $3.6 \mu 1 \mathrm{~min}^{-1}$ [7] to $30 \mu 1 \min ^{-1}$ [8] or even $100 \mu 1 \mathrm{~min}^{-1}$ [9] if the red blood cells are lysed. Inertial separation of rare cells from blood using microscale vortices was achieved at flow rates up to $10 \mathrm{ml} \mathrm{min}{ }^{-1}$ for 5 to 40 times diluted whole blood [10-12]. Other methods include deterministic lateral displacement that has a whole-blood throughput of $0.018 \mu 1 \mathrm{~min}^{-1}$ [13] and hydrodynamic filtration with a whole-blood throughput of $2 \mu 1 \mathrm{~min}^{-1}$ [14]. For acoustic separation devices the whole-blood throughput commonly varies from $5 \mu 1 \mathrm{~min}^{-1}$ for the enrichment of mononuclear cells [15] to over $20 \mu \mathrm{lmin}^{-1}$ for the separation of different leukocyte subpopulations [5].

Several strategies exist to increase the whole-blood, i.e., sample, throughput in addition to increasing the sample flow rate, for example, multiple channels can be built on a single lab-on-a-chip device $[8,16]$. In principle, microfluidic devices can be driven at increased flow rates as long as the flow remains in the Stokes regime, which is defined by a vanishing small Reynolds number. The Reynolds number, given by $\rho v d / \eta$, with the fluid density $\rho$, dynamic viscosity $\eta$, characteristic velocity $v$, and length scale $d$, gives an estimate for the ratio between inertial and viscous forces. Due to the small characteristic length scale and the relatively low velocities in microfluidic systems, the fluid dynamics are often well described using the so-called Stokes approximation and neglecting all inertial forces. 
However, at higher flow rates the inertial forces can play a significant role in the motion of the fluid even if the flow remains nonturbulent. The inertial effects are not always detrimental, some applications such as inertial focusing [7-9] actively rely on such forces. Nonetheless, the inertia forces will change the flow dynamics and it is not yet clear to what extent an application such as acoustophoresis, which is designed for the Stokes regime, might be impacted by inertia effects at high flow rates.

In this study, we investigate to what extent the sample throughput can be increased using a previously presented acoustophoresis microchip [15] by altering the sample flow rate or changing the splitting ratio between the sample and the sheath buffer. Here, the inertial effects on the particle trajectories through the main separation channel and the two trifurcation forks at both ends of the separation channel are studied theoretically and experimentally for a wide range of flow settings. Theoretical calculations of the critical flow settings for the particle separations are found to match the experimental outcome, providing means of future in silico chip design optimization.

\section{MATERIALS AND METHODS}

\section{A. Experimental setup}

The acoustofluidic chip is manufactured by Micronit (Enschede, Netherlands) using deep reactive ion etching in silicon and covered with a glass lid. Briefly, the microfluidic chip has a sample inlet and prefocusing channel (length $\times$ width $\times$ depth; $26 \mathrm{~mm} \times 300 \mu \mathrm{m} \times 150 \mu \mathrm{m}$ ), followed by a $v$-shaped flow splitter and sheath buffer inlet (inlet fork) with a subsequent main separation channel (43 $\mathrm{mm} \times 375 \mu \mathrm{m} \times 150 \mu \mathrm{m})$ and lastly, an outlet trifurcation (outlet fork) with a center outlet and a common outlet for the two side branches. The prefocusing channel is actuated with a lead zirconium titanate piezoelectric ceramic transducer (PZT) at $5 \mathrm{MHz}$, which generates two pressure nodes at channel mid height and at the lateral positions $x=93.75 \mu \mathrm{m}$ and $x=-93.75 \mu \mathrm{m}$ (see Fig. 1). The purpose of the prefocusing channel is to position all the sample particles into the same lateral $(x)$ position before they enter the main separation channel. The main separation channel is actuated by an additional PZT transducer at $2 \mathrm{MHz}$, which generates a pressure node at the channel mid height and $x=0 \mu \mathrm{m}$ (see Fig. 1). The transducers are actuated with a dual function generator (AFG 3022B, Tektronix INC, Bracknell, UK) connected to an in-house build power amplifier and the applied voltages and frequencies are measured with an oscilloscope (TDS 1002, Tektronix INC, Bracknell, UK). The temperature of the chip is kept between 25.0 to $25.5^{\circ} \mathrm{C}$ with a Peltier controller (TC0806RS232, CoolTronic, Beinwil am See, Switzerland) and is monitored by a PT100 resistance temperature detector. The flow rates and splitting ratios are controlled by a LabVIEW

(a)
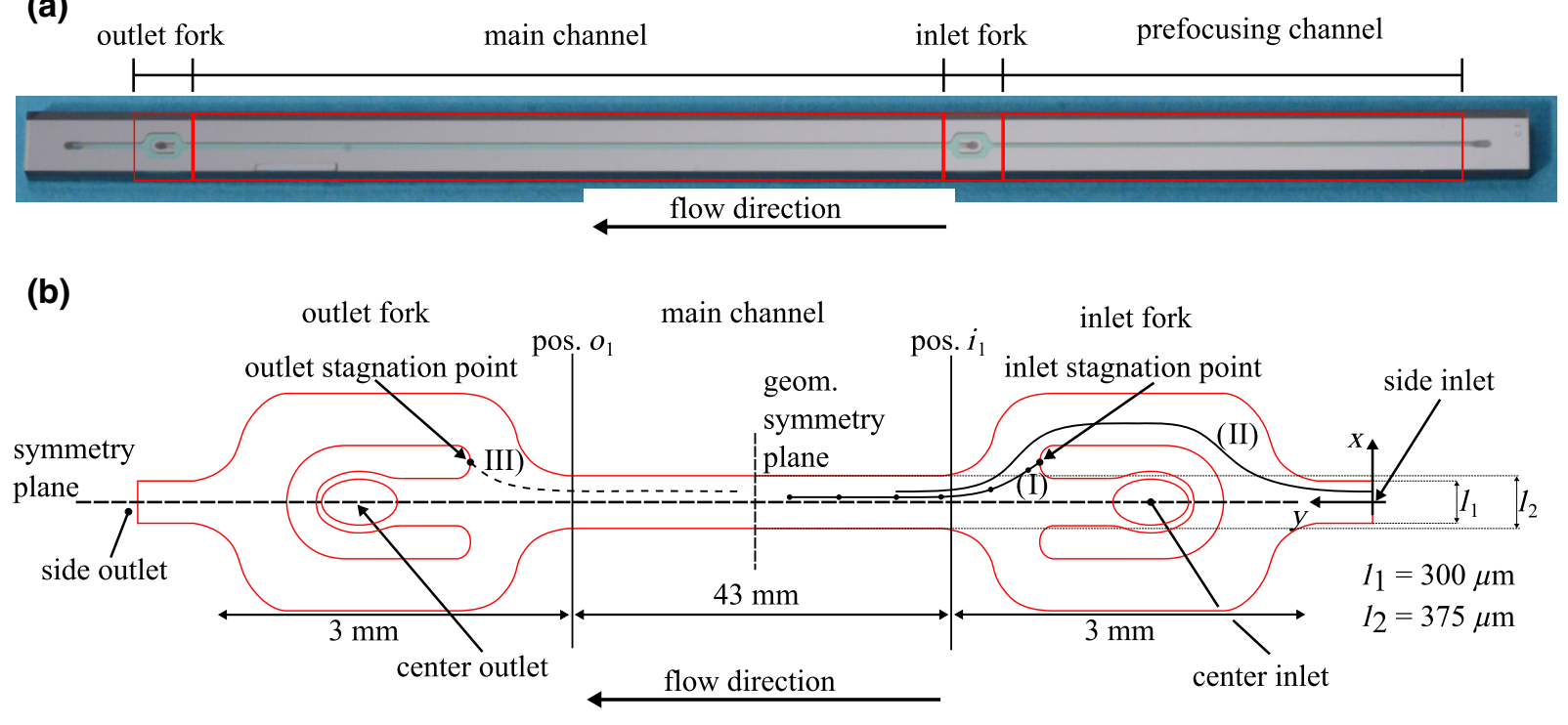

FIG. 1. Photograph (a) and sketch (b) of the device. The device consists of four central parts, i.e., the prefocusing channel, the buffer inlet fork, the main separation channel, and the outlet fork. The input sample containing suspended particles enters the device through the side inlet, after being acoustically prefocused in two nodes at mid height of the prefocusing channel, and the sheath buffer enters through the center inlet. The channel depth is $150 \mu \mathrm{m}$. The setup has two symmetry planes, where the symmetry plane along the $y$ axis is a true symmetry plane that is used to reduce the model size. The symmetry plane parallel to the $x$ axis is only a geometrical symmetry plane and could not be used to reduce the size of the model. Three types of streamlines, all collected at channel mid height, are of interest: the inlet stagnation streamline (I) starts at the inlet-fork wall, the particle streamline (II) follows the path of the particles, and the outlet stagnation streamline (III) ends at the outlet-fork wall. 




FIG. 2. Image of the inlet fork at $r_{\text {in }}=0.65, r_{\text {out }}=0.35$, and $Q_{\text {tot }}=1000 \mu 1 \mathrm{~min}^{-1}$. The red outline is drawn in Inkscape and forms the basis of the numerical model. The geometrical scale is given by the $375 \mu \mathrm{m}$ width of the main channel (left). The drawing of the channel contour (red) is imported into COMSOL and extruded into the third dimension to create the model geometry. The geometry is the same for the models of the inlet and outlet forks. The only differences between the models are the boundary conditions that are applied to boundaries 1,2 , and 3 . In the model for the inlet fork, boundaries 2 and 3 are modeled as inlets, while in the model for the outlet fork boundaries 2 and 3 are modeled as outlets. Similarly, the boundary is modeled as an outlet or inlet in the models for the inlet and outlet fork, respectively.

software and a pressure driven system (FESTO, VEMA 563303 VALVE TERMINAL, Esslingen, Germany). The flow rate is measured by three flow sensors (Liquid Flow Meter SLI-1000, SENSIRION, Staefa, Switzerland) at the center inlet and at the two outlets. The total flow rate is set between 200 and $1000 \mu 1 \mathrm{~min}^{-1}$.

The device, shown in Fig. 1, consists of four central parts parts, i.e., the prefocusing channel, the buffer inlet fork, the main separation channel, and the outlet fork. The sample enters the device through the side inlet, and the sheath buffer enters through the center inlet at the inlet fork. The splitting ratio $r_{\text {in }}$ at the inlet fork is defined as the flow rate at the side inlet $Q_{s \text {, in }}$ divided by the total flow rate $Q_{\text {tot }}=Q_{s, \text { in }}+Q_{c \text {,in }}$, formally, $r_{\text {in }}=Q_{s, \text { in }} / Q_{\text {tot }}$, where $Q_{c \text {,in }}$ denotes the center flow rate. The fluid can exit the device at either the center or the side outlet. The splitting ratio at the outlet fork is defined analogously to the splitting ratio at the inlet fork, formally, $r_{\text {out }}=Q_{s, \text { out }} / Q_{\text {tot }}$. In our standard setting, the splitting ratios at the inlet and outlet forks are chosen such that the volume entering at the side inlet and the volume exiting the device at the center outlet are equal, formally this yields $r_{\text {in }}+r_{\text {out }}=1$. Several different splitting ratios are investigated, varying between $r_{\text {in }}=0.10$ and $r_{\text {out }}=0.90$ to $r_{\text {in }}=0.70$ and $r_{\text {out }}=0.30$.

\section{B. Confocal measurements}

The measurements are performed with a confocal microscope (Eclipse Ti2, Nikon, Tokyo, Japan) equipped with a spinning disk unit (X-Light V3, Crest, Rome, Italy) and a CMOS camera (Prime 95B, Teledyne Photometrics, Tucson, AZ). Fluorescein isothiocyanate (FITC) labeled
Ficoll (Polysucrose 400-fluorescein isothiocyanate conjugate, Sigma-Aldrich Sweden AB, Stockholm, Sweden) is added to the milli-Q water with a mass concentration of $0.07 \%$. Blue-fluorescent excitation light is illuminated from a laser diode with a peak wavelength of $488 \mathrm{~nm}$, and a standard fluorescence filter cube is used with an excitation passband from 475 to $495 \mathrm{~nm}$ and an emission passband from 510 to $531 \mathrm{~nm}$. An objective lens with $10 \times$ magnification and 0.3 numerical aperture is used, and this configuration provides an optical slice thickness of $5.7 \mu \mathrm{m}$. For each scan in height direction, 55 images are acquired with a step size of $3 \mu \mathrm{m}$, leading to a measurement volume of $1.83 \times 1.83 \times 0.215 \mathrm{~mm}^{3}$ with the refractive-index correction.

\section{Particles and buffer medium}

Fluorescent $5.19 \mu \mathrm{m}$ in diameter and/or $7.81 \mu \mathrm{m}$ polystyrene particles (PS-FluoGreen, microparticles $\mathrm{GmbH})$ are suspended in FACS buffer (1xPBS, 1\% FBS, $2 \mathrm{mM}$ EDTA) and run through the chip from the side inlet.

\section{Numerical setup}

A three-dimensional finite-element model is set up in COMSOL Multiphysics to investigate the hydrodynamic flow fields theoretically. The domain geometry is extracted from pictures taken during the experiment. The setup has two symmetry planes, where the symmetry plane along the $y$ axis is a true symmetry plane and is exploited to reduce the model size. In the following, we consider only the upper half of the device in our analysis. The symmetry plane parallel to the $x$ axis, is only a geometrical symmetry plane that cannot be used to reduce the size of the numerical model.

Three types of streamlines, all collected at channel mid height, are investigated: the particle streamline of the prefocused particles, the stagnation streamline at the inlet fork and the stagnation streamline at the outlet fork. The particle streamline [Fig. 1(b)] follows the path of the particles. The lateral $(x)$ position of the particle streamline is determined by the $\lambda$ and $\lambda / 2$ focussing in the width and height direction of the prefocussing channel. From the experiments, we find that the particle streamline starts at a lateral position $x=75 \mu \mathrm{m}$ at the inlet of the inlet fork and then passes through the system. The position of the particle streamlines is measured from the photographs. In Sec. IV, we show theoretically that the particles follow the streamlines almost perfectly. The inlet stagnation streamline (Fig. 1I) starting at the inlet-fork wall (inlet stagnation point) and separates the flow entering from the side inlet from the flow entering through the center inlet at channel mid height. The outlet stagnation streamline (Fig. 1III) starts at the wall of the outlet fork (outlet stagnation point) 
(a)

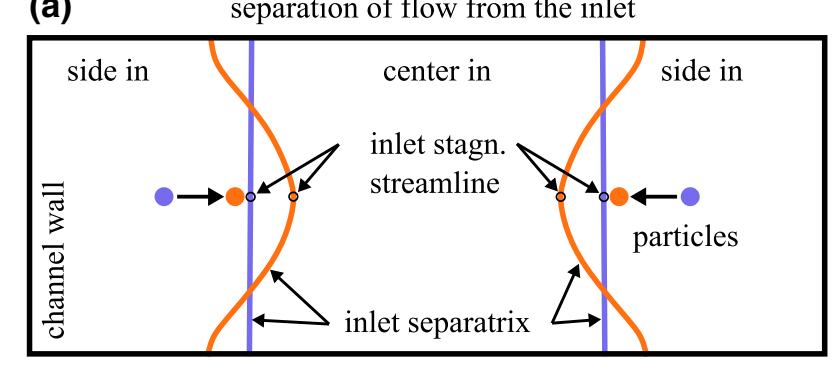

Inertial effect on the separatrix

separation of flow from the inlet

(b)

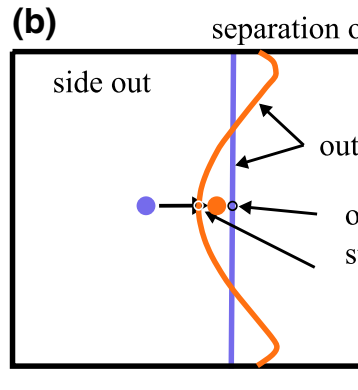

Stokes flow

high flow rate
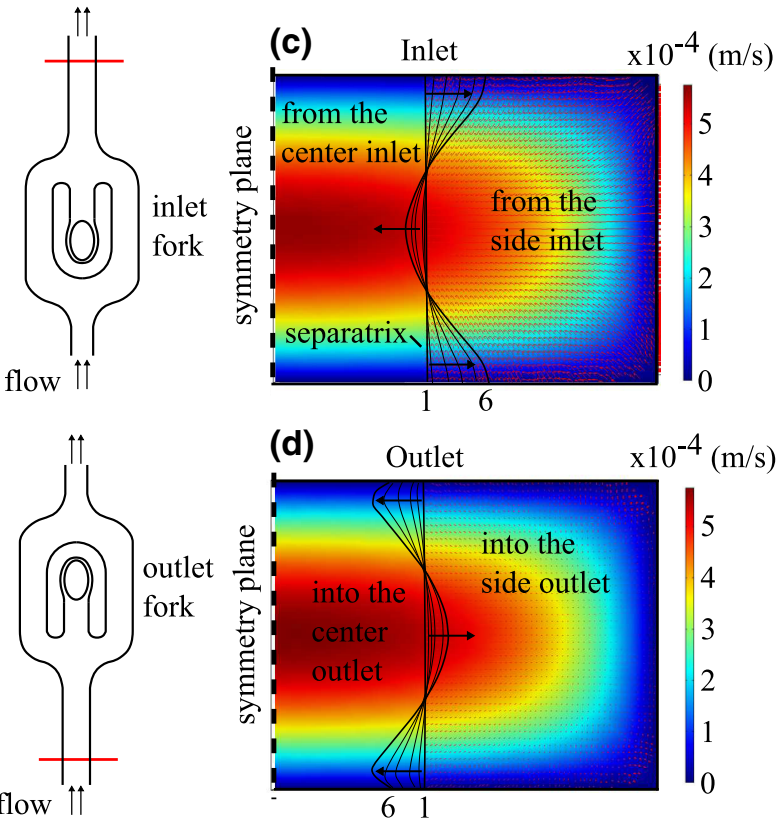

FIG. 3. Sketch of the underlying mechanism to the spillover effect. The separatrix is a (virtual) surface that separates fluid volumes. In our setup two different separatrices are of interest: the inlet separatrix separates fluid that comes from the side inlet from fluid that enters via the center inlet [(a),(c)] and the outlet separatrix separates fluid exiting through the side outlet from fluid exiting through the center outlet [(b),(d)]. The mid height of the separatrix is denoted the stagnation streamline (at mid height). At high flow rates the inertia forces lead to a deformation of the separatrices. At the inlet, the deformation displaces the prefocused particle stream closer to the center of the channel. At the outlet, the deformation of the outlet separatrix moves its mid height closer to the channel walls. At high enough flow rates and specific splitting ratios the prefocused particle stream exits through the center outlet. COMSOL simulation of the shape of the inlet separatrix (c) and outlet separatrix (d). Six different flow rates from 1 to $1000 \mu 1 \mathrm{~min}^{-1}$ are used in the simulation and annotated by 1 to 6 , respectively. The background shows the velocity amplitude in the main channel for a total flow rate of $1 \mu 1 \mathrm{~min}^{-1}$. The inlet fork and outlet fork are shown in the middle at the top and bottom panels, respectively. The red cut indicates the part of the main channel that is used for (c),(d).

and separates the flow into fluid exiting via the side or center outlet at channel mid height.

A sketch of the numerical setup is shown by the red outline in Fig. 2. An image of the chip inlet fork is taken and an overlay is drawn in Inkscape. The overlay is then imported into COMSOL and extruded into the third dimension. We exploit the symmetry of the setup to reduce the model size by half, thus rendering the computations more efficient. The water (density $\rho=998 \mathrm{~kg} \mathrm{~m}^{-3}$ and dynamic viscosity $\eta=1.01 \mathrm{mPa} \mathrm{s}$ ) domain is modeled as stationary incompressible laminar flow. The equations describing the fluid dynamics in this case are well known $[17,18]$, and given by the conservation of (linear) momentum

$$
\rho(\mathbf{u} \cdot \nabla) \mathbf{u}=-\nabla p+\eta \nabla^{2} \mathbf{u},
$$

and the conservation of mass

$$
\rho \nabla \cdot \mathbf{u}=0,
$$

where we use the velocity field $\mathbf{u}$, pressure field $p$, the fluid dynamic viscosity $\eta$, and density $\rho$. The equations are solved using the laminar flow module in COMSOL, which allows inclusion of weakly nonlinear inertial effects for nonturbulent flows.

The geometry is the same for both the inlet and outlet forks, however, the boundary conditions at boundaries 1, 2 , and 3 are chosen accordingly, see Fig. 2. At the inlet fork, $b$ and $c$ are fully-developed-flow inlets with flow rates $0.5 \times\left(1-r_{\text {in }}\right) \times Q_{\text {tot }}$ and $0.5 \times r_{\text {in }} \times Q_{\text {tot }}$, respectively. At the inlet-fork boundary, $a$ is modeled as a pressure release outlet. Analogously, in our model of the outlet fork, boundaries 2 and 3 were modeled as fullydeveloped-flow outlets with flow rates $0.5 \times\left(1-r_{\text {out }}\right) \times$ $Q_{\text {tot }}$ and $0.5 \times r_{\text {out }} \times Q_{\text {tot }}$, respectively. Note that the factor 0.5 is introduced because only half of the geometry is included in the numerical model. A nonslip boundary condition is applied to all the outer boundaries, while the symmetry plane is modeled by a symmetry boundary condition. 

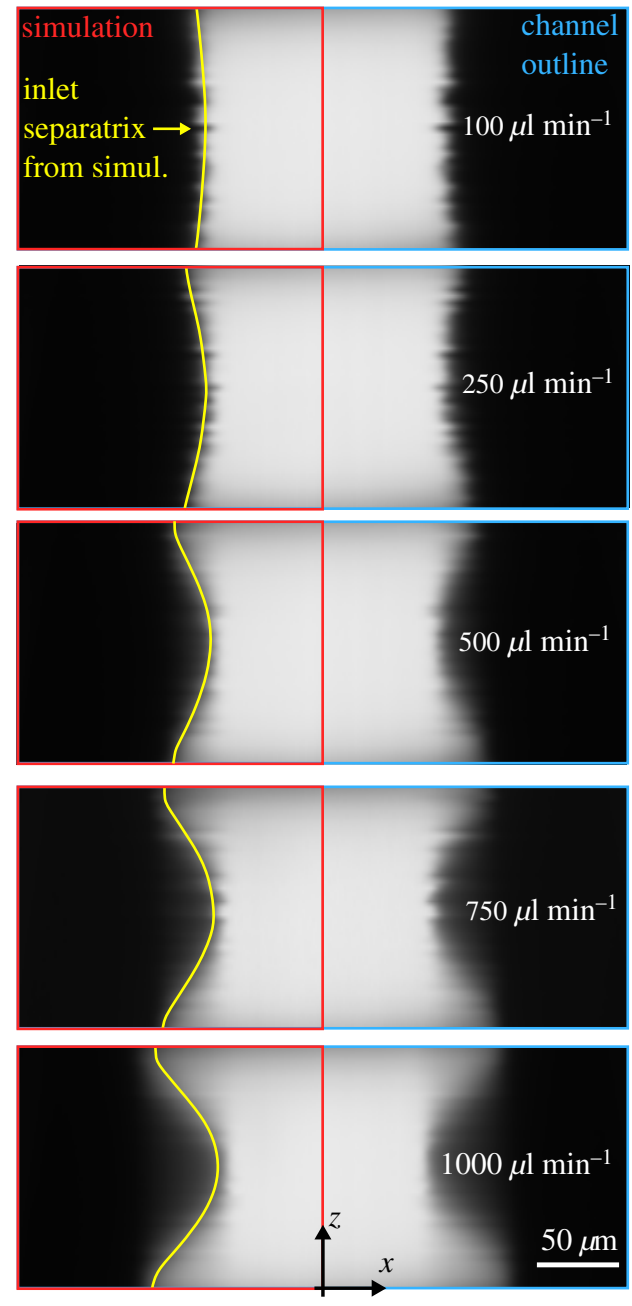

FIG. 4. The inlet separatrix (yellow) computed in the simulation agrees well with the confocal measurements (black and white) taken close to the inlet fork. The channel height extends over the bright white area and is sketched by the red and blue outline. The red outline is the half-channel cross section from the simulation, the yellow outline of the separatrix is computed in the simulation, the black and white are confocal measurements and the blue outline indicates the channel cross section. Water containing FITC-labeled Ficoll entered the channel through the center inlet at a splitting ratio of $r_{\text {in }}=r_{\text {out }}=0.5$ and total flow rates ranging from 100 to $1000 \mu 1 \mathrm{~min}^{-1}$.

\section{RESULTS}

\section{A. Particle trajectories}

In a first step, we check if the particles follow the streamlines accurately and if they experience any lateral displacement in the main channel, when the main acoustic field is turned off. We investigate if the particle trajectories experience an additional lateral drift in the main channel, linked to a particle-wall interaction (walllift force), which has been extensively investigated in the literature [19-21]. Although, the particles might experience a small force due to the particle-wall interactions, the displacements due to the particle-wall interactions are found to be in the order of $10^{-3}$ times the particle radius (Appendix A 1). It is thus concluded that the lateral positions at $i_{1}$ and $o_{1}$, see Fig. 1, could be approximated to be equal and the main channel is hence forth neglected in the continued theoretical investigation.

Thereafter, we study if the particles followed the streamlines of the flow, or if the centrifugal drift moves the particles across streamlines. Appendix A 4 analytically shows that the centrifugal drift of the particles is in the order of a few percent of the particle diameter, and therefore it can be assumed that the particles follow the streamlines with good accuracy.

\section{B. The spillover effect}

In this section, we theoretically investigate the physical mechanism that drives the spillover effect. For certain settings of the inlet and outlet splitting ratios, we observe in the experiments that the prefocused particle stream exits through the side outlet at a low total flow rate (Stokes flow) but when increasing the total flow rate above a critical value the prefocused particle stream exited through, i.e., spilled over into, the center outlet. This effect is observed without applying any acoustic field to the main channel and while keeping the splitting ratios constant. The flowrate-dependent spilling of particles, coined "the spillover effect," is detrimental as it makes an acoustic separation of different particles (or cells) inefficient or even impossible at certain flow settings.

First we introduce the concept of a flow separatrix: the separatrix is a (virtual) surface that separates fluid volumes. Amini et al. [22] have shown that the separatrix can deform under the action of inertial forces. Here, we consider two different separatrices, the first, denoted inlet separatrix, separates fluid entering through the side inlets from fluid entering through the center inlet, the second, denoted outlet separatrix, separates fluid exiting to the side outlets from fluid exiting through the center outlet. The separatrices are surfaces, however, they reduce to lines if we consider them at mid height only. The streamlines that are the inlet separatrix and outlet separatrix collected at mid height are (here) denoted by inlet stagnation streamline and outlet stagnation streamline, respectively. At high flow rates, the inertial forces start deforming the flow field and the separatrices, which are flat surfaces in Stokes flow, as sketched in Figs. 3(a) and 3(b). At the inlet, the deformation of the inlet separatrix drives the prefocused particle stream closer to the channel center [Fig. 3(a)]. At the outlet, the outlet separatrix deforms such that particles closer to the side walls are collected into the center outlet, as shown at the bottom panel of Fig. 3(b). 

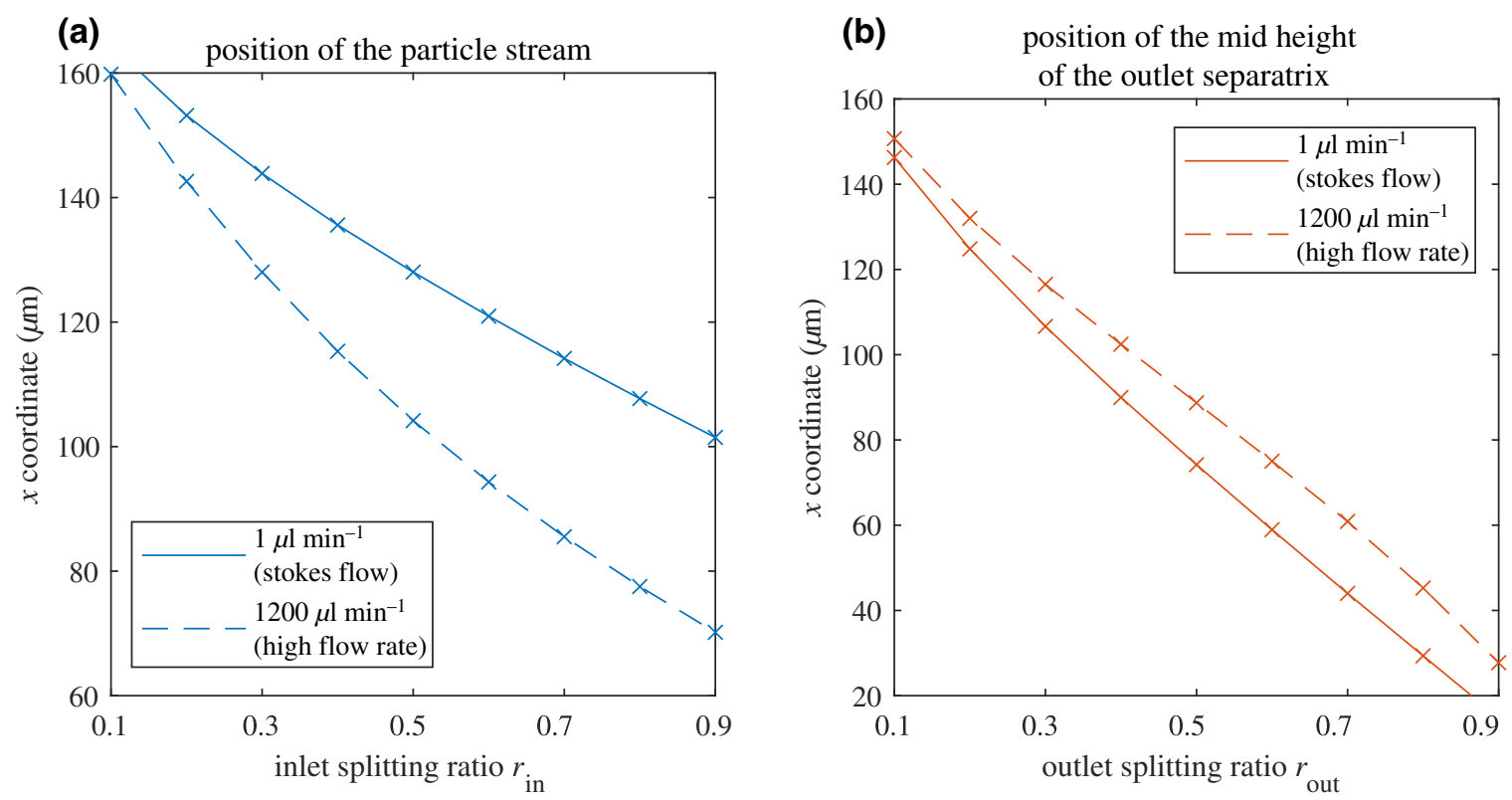

FIG. 5. The lateral distance to the channel center ( $x$ position) of the particle stream (a) and mid height of the outlet separatrix (b) from the upper half of the device. If settings, i.e., total flow rate and splitting ratio at the inlet and outlet, are chosen such that the particle stream is above, i.e., has a larger $x$ coordinate than, the outlet separatrix then the particles will exit through the side outlet. If their lateral $(x)$ position is below the outlet separatrix then the particles exit through the center outlet. The inertia-induced spillover occurs when the prefocused particles exit through the side outlet at Stokes flow but then exit through the center channel at an increased total flow rate, while the inlet and outlet splitting ratios are kept constant. Only settings where the prefocused particle stream exits through the side outlet can be used for the acoustic separation. The cross markers denote the data points that are generated by the simulation and used as the basis for the line graphs.

For sufficiently high total flow rates, the deformation of the separatrices are so significant that the prefocused particle stream exits through the center outlet [Fig. 3(b)].

Figures 3(c) and 3(d) show the numerically computed inlet and outlet separatrix. Six different flow rates are applied to compute the separatrices: $1,100,250,500,750$, and $1000 \mu 1 \mathrm{~min}^{-1}$, annotated from 1 to 6 , respectively. The splitting ratios are chosen to be 0.5 both at the inlet and outlet, i.e., $r_{\text {in }}=r_{\text {out }}=0.5$.

To show the deformation of the inlet separatrix experimentally, we take confocal measurements of the channel cross section close to the inlet fork. At various flow rates and a splitting ratio of $r_{\text {in }}=r_{\text {out }}=0.5$ we pump milli$\mathrm{Q}$ with 0.7 mass percent FITC labeled Ficoll through the channel inlet, while we use standard (nonfluorescent) milli-Q in the side inlets. The overlay of the numerical results on top of the experimental results are shown in Fig. 4. The red outline denotes the channel half cross section in the numerical simulation. The yellow outline of the separatrix is computed numerically. The blue channel outline is the measured channel cross-section outline based on the pixel size. Our theoretical prediction of the shape and position of the inlet separatrix agrees well with the confocal measurements. If a spillover occurs, it is (a)

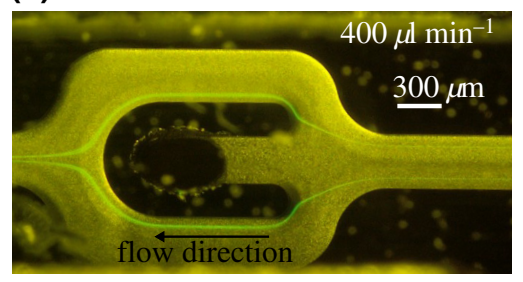

(b)

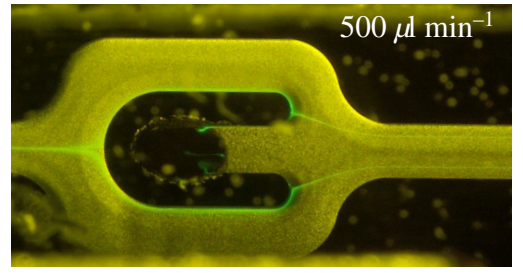

(c)

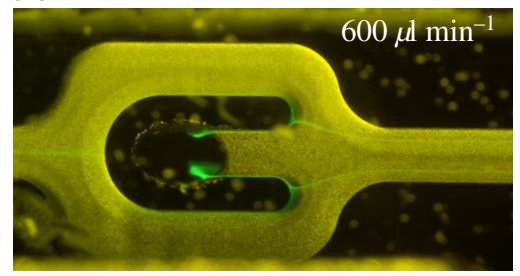

FIG. 6. The particle trajectories of fluorescent 5.19- $\mu \mathrm{m}$ diameter polystyrene beads in FACS buffer are shown at the outlet for splitting ratios $r_{\text {in }}=0.65$ and $r_{\text {out }}=0.35$. (a) At $400 \mu 1 \mathrm{~min}^{-1}$ all the particles exited through the side outlet. (b) A few prefocused particles spilled into the center outlet at $500 \mu 1 \mathrm{~min}^{-1}$. The spillover effect increased with increased flow rate and can easily be observed at $600 \mu \mathrm{l} \mathrm{min}-1$ as shown in (c). 
(a)

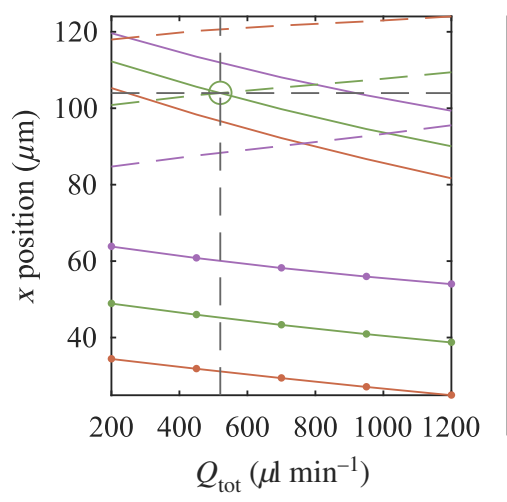

(b)

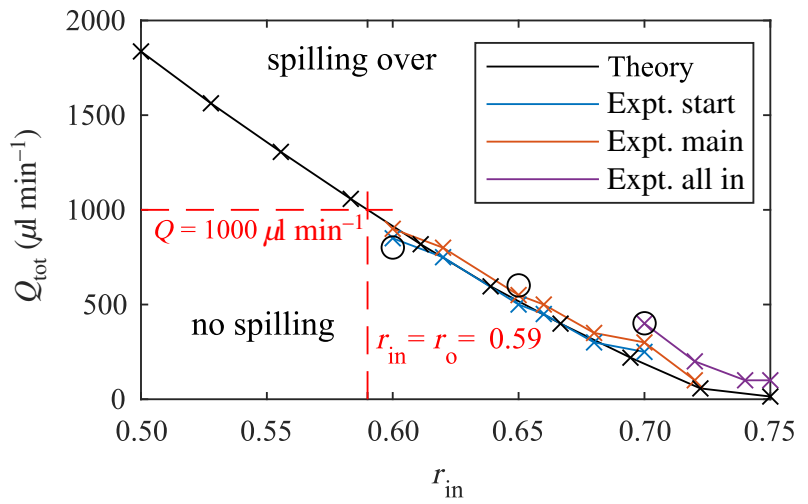

FIG. 7. Panel (a) shows the theoretical lateral deviation from the center of the channel ( $x$ position) for the particle streamline (solid), the stagnation streamline at the inlet (solid with dot, $\left.S_{\text {in }}\right)$ and outlet (dashed, $\left.S_{\text {out }}\right)$ for three splitting ratios $\left(r_{\text {in }}=0.55,0.65,0.75\right.$ with $r_{\text {in }}+r_{\text {out }}=1$ ). Panel (b) shows the critical total flow rate $Q_{\text {tot }}$ as a function of the inlet splitting ratio $r_{\text {in }}, r_{\text {in }}+r_{\text {out }}=1$. The solid black line represents the theoretical prediction obtained from our finite-element model, and the stacked crossmark lines represent the experimental data. In our experimental setup, the maximal total flow rate is $Q_{\mathrm{tot}}=1000 \mu \mathrm{min}^{-1}$, which leads to an optimal theoretical splitting ratio of $r_{\text {in }}=0.59$ and $r_{\text {out }}=0.41$. Note that in experiments it is advised to avoid close-to-critical flow rates and splitting ratios, due to imperfections in the setup. In the experiments the spill-over phenomenon does not happen instantaneously, but rather as a continuous effect, and therefore the data is separated into three categories, as shown in Fig. 9: (1) a few particles spilling into the main channel marks the onset of the spillover, denoted by Exp. start; (2) the spilling over is clear and particles exit both via the side and center outlet (Exp. main); (3) all the particles exit via the center outlet (Exp. all in). The circles in (b) correspond to the settings from Fig. 10 (d) $\left(r_{\text {in }}=0.60, r_{\text {out }}=0.40,800 \mu 1 \mathrm{~min}^{-1}\right)$, (e) $\left(r_{\text {in }}=0.65, r_{\text {out }}=0.35,600 \mu 1 \mathrm{~min}^{-1}\right)$, and (f) $\left(r_{\text {in }}=0.70, r_{\text {out }}=0.30\right.$, $400 \mu 1 \mathrm{~min}^{-1}$ ). The black cross markers correspond to data points obtained from the simulation and the blue, red, and violet cross markers correspond to experimentally obtained datapoints.

ultimately determined by the relative $x$ position of the particle stream with respect to the $x$ position of the outlet stagnation streamline. Note that the mid height of the outlet separatrix coincides with the position of the outlet stagnation streamline. The lateral $(x)$ position of the particle stream is a function of the total flow rate and the splitting ratio at the inlet $r_{\text {in }}$, while the position and shape of the outlet separatrix are a function of the total flow rate and the outlet splitting ratio $r_{\text {out }}$. For the following analysis, we exploit the symmetry and limit our investigation to the upper half of the device $x>0$. The lateral $(x)$ position of the particle streamline is collected at the position $i_{1}$ (see Fig. 1) and the mid height of the outlet separatrix is collected at position $o_{1}$. Figure 5 shows the $x$ coordinate of the particle streamline on (a) and the $x$ position of the outlet stagnation streamline (mid height of the outlet separatrix) on (b) for total flow rates of 1 and $1200 \mu 1 \mathrm{~min}^{-1}$ as a function of the inlet and outlet splitting ratio, respectively. To avoid a spillover, the inlet splitting ratio $r_{\text {in }}$, outlet splitting ratio $r_{\text {out }}$, and total flow rate should be chosen such that the $x$ coordinate of the particle stream is larger than the $x$ coordinate of the mid height of the outlet separatrix. The result allows investigation of the impact of the inlet and outlet splitting ratios separately, while we restrict the experimental analysis in this work to $r_{\text {in }}+$ $r_{\text {out }}=1$. At increased flow rates, the particle stream moves closer to the center of the channel (smaller $x$ coordinate) while the outlet stagnation streamline (mid height of the outlet separatrix) moves closer to the sidewalls (larger $x$ coordinate). This means that the system becomes more prone to spilling over and the splitting ratios need to be selected carefully.

\section{Particle trajectories and the spillover effect}

The effect of inertial forces on the particle trajectories at high sample throughputs is investigated. At low flow rates, the prefocused polystyrene particles exited through the side outlet, but with increased flow rates inertial effects are observed as the particle trajectories are laterally displaced towards the center outlet. Finally, above specific flow settings, the particle trajectory spilled over from the side outlet branches into the center outlet. At the flow splitting ratio of $r_{\text {in }}=0.65$ and $r_{\text {out }}=0.35$, the particles spilled over from the side outlet into the center outlet at the outlet fork when the total flow rate is increased from 400 to $600 \mu 1 \mathrm{~min}^{-1}$, as shown in Fig. 6 .

Notably the effect is independent of the particle size, as particles of both 5.19 and $7.81-\mu \mathrm{m}$ diameter spilled over at the same critical flow rates and flow splitting ratios (see Appendix A 2). The effect is also independent of the acoustic prefocusing, as an additional increase in prefocusing voltage did not change the critical flow rates 


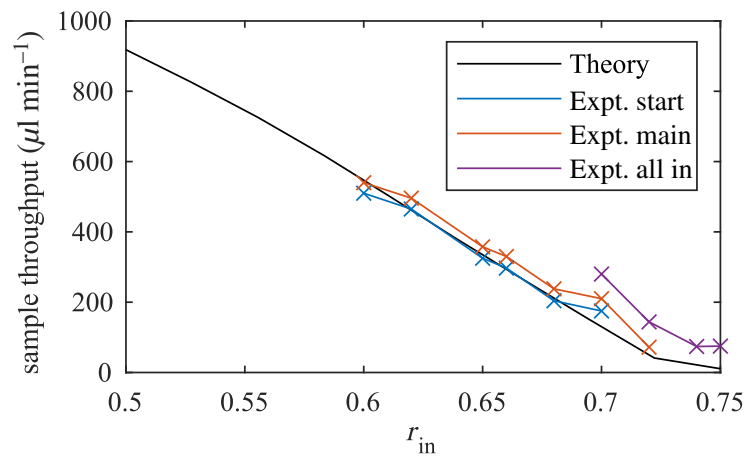

FIG. 8. The optimal settings for maximized sample throughput is limited by the maximal total flow rate supported by the system. In principle, the maximum theoretical sample throughput is achieved at the highest total flow rate with a splitting ratio $\left(r_{\text {in }}\right)$ closest to the theoretical limit. For the setup under study, the maximal flow rate is $1000 \mu \mathrm{lmin}^{-1}$, limited by the flow sensors in the setup, which gave an optimal theoretical splitting ratio of $r_{\text {in }}=0.59$ and $r_{\text {out }}=0.41$. The sample throughput is shown on the $y$ axis of the graph.

and flow splitting ratios (see Appendix A 3), which is in line with our theoretical modeling.

Figure 7(a) shows the theoretical lateral positions of the particle streamline (solid), the stagnation streamline at the inlet (solid with dot, $S_{\text {in }}$ ) and outlet (dashed, $S_{\text {out }}$ ) for three splitting ratios $\left(r_{\text {in }}=0.55,0.65,0.75\right.$ with $\left.r_{\text {in }}+r_{\text {out }}=1\right)$. For $r_{\text {in }}=0.65$ the particle streamline (solid green line) crosses the outlet stagnation streamline (dashed green line) between 400 and $600 \mu 1 \mathrm{~min}^{-1}$ indicating a spillover event. For $r_{\text {in }}=0.55$ the theory predicts that the maximal experimental flow rate of $1000 \mu 1 \mathrm{~min}^{-1}$ is not high enough to induce a spillover. For $r_{\text {in }}=0.75$ the theory predicts that the particles exit through the center channel already at Stokes flow. Figure 7(b) shows the critical total flow rate $Q_{\text {tot }}$ as a function of the inlet splitting ratio $r_{\text {in }}, r_{\text {in }}+r_{\text {out }}=1$. The black crossmark line is the theoretical prediction obtained from the finite-element model and the colored (blue, red, and violet) stacked crossmark lines represent the experimental data. Note that eight simulations using the bisection method are carried out in order to obtain one datapoint for the solid line. For a fixed splitting ratio, the critical flow rate is determined numerically with an accuracy of $10 \mu 1 \mathrm{~min}^{-1}$. Theoretically, the maximum sample throughput is achieved for the highest possible total flow rate $Q_{\text {tot }}$ with a flow ratio $\left(r_{\text {in }}\right)$ closest to the theoretical limit.

In the experiments, fluorescent $5.19 \mu \mathrm{m}$ polystyrene beads are run through the chip with acoustic prefocusing at $4.8 \mathrm{Mhz}$ and $12 \mathrm{~V}_{\mathrm{pp}}$ to obtain precise trajectories. The prefocusing moves and aligns the particles at the two pressure nodes in the prefocusing channel. Eleven different splitting ratios are investigated (using $r_{\text {in }}+r_{\text {out }}=1$ the used inlet splitting ratios $r_{\text {in }}$ are $0.50,0.55,0.60,0.62$, $0.65,0.66,0.68,0.70,0.72,0.74$, and 0.75 ) and the flow rate is increased from 100 to $1000 \mu \mathrm{min}^{-1}$ in steps of $50 \mu 1 \mathrm{~min}^{-1}$. Fluorescent images are captured over the outlet fork to visualize findings.

For this experimental setup, the maximal total flow rate, limited by the dynamic range of the flow sensors, is $1000 \mu 1 \mathrm{~min}^{-1}$. This yields a theoretical optimal splitting ratio of $r_{\text {in }}=r_{\mathrm{o}}=0.59$ and $r_{\text {out }}=0.41$, and a sample throughput of $590 \mu \mathrm{l} \mathrm{min}^{-1}$ (Fig. 7), if $r_{\text {in }}+r_{\text {out }}=1$ is respected. Figure 8 shows the maximal sample throughput as a function of the splitting ratio.

In the experiments, the particle stream had a finite width and as a result the spillover event occurred not instantly, but rather as a continuous process over a range of flow rates. Therefore, the spillover events are separated, as shown on (b) of Fig. 7, into three categories that are also illustrated in Fig. 9: (a) a few particles spilling into the center outlet marks the onset of the spillover, denoted by Exp. start; (b) the spilling over is now clear and particles exit both via the side and center outlet (Fig. 7 Exp. main); (c) all the particles exit via the center outlet (Fig. 7 Exp. all in). The numerical results are in good agreement with the Exp. start and Exp. main results (Figs. 7 and 8). The slight offset to the Exp. all in curve is expected, as the center and not the outer boundary of the particle stream is chosen as the starting point of the particle streamline in the numerical investigation. Further, we investigate the sensitivity of the spillover effect on the width of the particle theoretically. In Fig. 11, the particle streams are shown at the outlet fork for $r_{\text {in }}=0.65$ and $r_{\text {out }}=0.35$ for five different flow
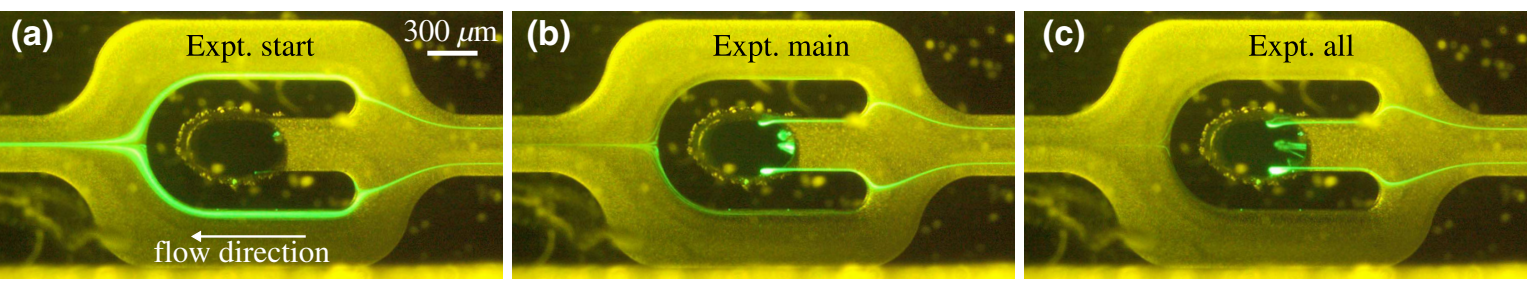

FIG. 9. Spillover into the center outlet for $5.19-\mu \mathrm{m}$ polystyrene particles in FACS buffer. For splitting ratios of $r_{\text {in }}=0.70$ and $r_{\text {out }}=0.30$ the spillover started (Exp. start) at (a) $250 \mu \mathrm{l} \mathrm{min}^{-1}$, could clearly be seen (Exp. main) at (b) $300 \mu 1 \mathrm{~min}^{-1}$ and (c) $400 \mu 1 \mathrm{~min}^{-1}$ generated a complete spillover (Exp. all) into the center outlet. 
TABLE I. Numerically computed critical flow rate for a splitting ratio of $r_{\text {in }}=0.65$ and $r_{\text {out }}=0.35$ and various values of fluid density and dynamic viscosity.

\begin{tabular}{|c|c|c|c|c|c|c|c|}
\hline Settings & a & $\mathrm{b}$ & $\mathrm{c}$ & d & $\mathrm{f}$ & $\mathrm{g}$ & $\mathrm{h}$ \\
\hline Density $\rho\left(\mathrm{kg} \mathrm{m}^{-3}\right)$ & 1000 & 1000 & 1000 & 1050 & 950 & 1050 & 950 \\
\hline Dyn. viscosity $\eta$ (Pa s) & 1.01 & 1.06 & 0.96 & 1.01 & 1.01 & 1.06 & 0.96 \\
\hline Crit. tot. flow rate $Q_{\text {tot,crit }}\left(\mu 1 \mathrm{~min}^{-1}\right)$ & 510 & 536 & 493 & 493 & 536 & 510 & 510 \\
\hline
\end{tabular}

rates. The more transparent colored particle streams are calculated by perturbing the initial lateral $(x)$ position of the particle stream before the inlet fork by $\pm 5 \mu \mathrm{m}$.

Furthermore, the sensitivity of the spillover effect on smaller variations of the material properties of the buffer is studied theoretically. The critical total flow rate for a splitting ratio of $r_{\text {in }}=0.65$ and $r_{\text {out }}=0.35$ is numerically computed, while varying both the fluid density and dynamic viscosity by $\pm 5 \%$. The results of the sensitivity study are summarized in Table I. It can be observed that keeping the Reynolds number constant, i.e., keeping the ratio between density and dynamic viscosity constant, gives a constant critical total flow rate.

\section{Particle separation efficiency with increased flow rate}

Particles with different acoustic mobility migrate towards the central pressure node at different velocities $[4,15,23]$. In the Stokes regime the setup can be tuned such that larger particles with higher acoustic mobility exit the chip through the center outlet, while smaller particles with lower acoustic mobility exit through the side outlet. To this end, larger particles are moved below the outlet stagnation streamline while smaller particles stay above the outlet stagnation streamline at the position $o_{1}$, see Fig. 1. However, when the setup is driven beyond the critical flow rate and splitting ratio, a breakdown of the separation efficiency is expected.

Two sizes of fluorescent polystyrene particles $(5.19 \mu \mathrm{m}$, red and $7.81 \mu \mathrm{m}$, green) are mixed in FACS buffer and run through the side inlet of the acoustofluidic chip. Six different splitting ratios (using $r_{\text {in }}+r_{\text {out }}=1$ the inlet splitting ratios $r_{\text {in }}$ are $0.30,0.40,0.50,0.60,0.65$, and 0.70 ) and five flow rates for each ratio $(200,400,600,800$, and $1000 \mu 1 \mathrm{~min}^{-1}$ ) are investigated. Here, the prefocusing amplitude is constant at $10 \mathrm{~V}_{\mathrm{pp}}$, and the piezoelectric transducer attached under the main separation channel is tuned for each splitting ratio and flow-rate setting to achieve separation of the two bead sizes.

The separation collapses when the setup is driven beyond the critical flow rate and splitting ratio as all the particles exited through the center outlet regardless of applied acoustic amplitude and the particles' respective acoustic mobility. Figure 10 shows the inertia-induced breakdown of the separation efficiency of the particles. For
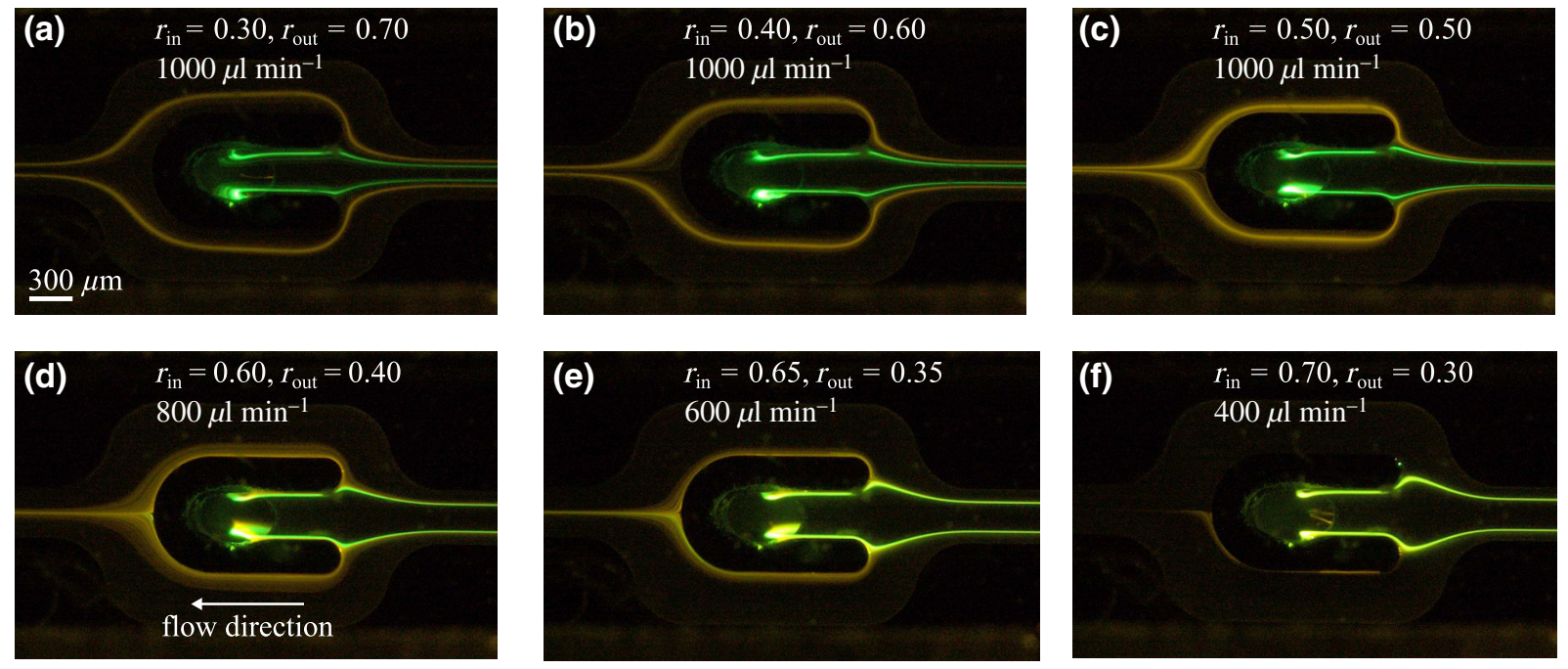

FIG. 10. Separation at the outlet fork of 5.19- $\mu \mathrm{m}$ (red) and 7.81- $\mu \mathrm{m}$ (green) polystyrene beads at different splitting ratios and flow rates. For all the experiments the splitting ratios at the inlet and outlet fork are coupled via $r_{\text {in }}+r_{\text {out }}=1$. For an inlet splitting ratio $\left(r_{\text {in }}\right)$ below 0.60 it is possible to separate the different sized beads even for the maximal total flow rate of $Q_{\text {tot }}=1000 \mu 1 \mathrm{~min}^{-1}$. For inlet splitting ratios $r_{\text {in }}$ of 0.60 or above, critical flow rates are found where it is impossible to separate the different sized beads. Successful separations are shown in (a)-(c), whereas (d)-(f) show failure to separate the particles. 
(a)

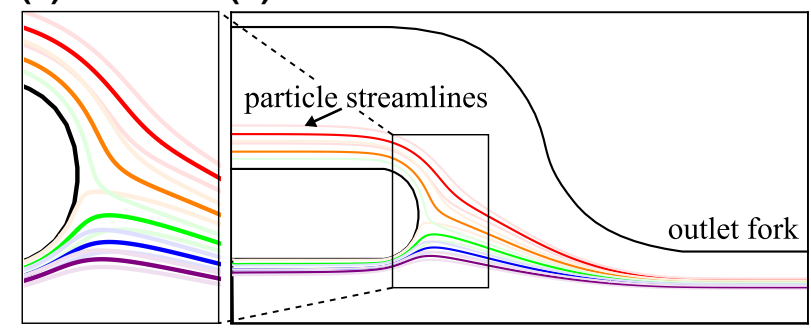

FIG. 11. Streamlines at the outlet fork for $r_{\text {out }}=0.35$ at different overall flow rates $Q$ : (red) $Q=200 \mu \mathrm{min}^{-1}$, (orange) $Q=400 \mu 1 \mathrm{~min}^{-1}$, (green) $Q=600 \mu 1 \mathrm{~min}^{-1}$, (blue) $Q=$ $800 \mu 1 \mathrm{~min}^{-1}$, and (purple) $Q=1000 \mu \mathrm{l} \mathrm{min}^{-1}$. The bands are given by a small perturbation of $\pm 5 \mu \mathrm{m}$ in the initial lateral $(x)$ starting position of the particle stream before the inlet fork.

an inlet splitting ratio $\left(r_{\text {in }}\right)$ below 0.60, Figs. 10(a)-10(c), it is possible to tune the acoustics and separate the different sized particles even at the maximal total flow rate of $Q_{\text {tot }}=$ $1000 \mu 1 \mathrm{~min}^{-1}$ [Figs. 10(a)-10(c)]. For inlet splitting ratios $\left(r_{\text {in }}\right)$ of 0.60 or above, critical flow rates are found where it is impossible to obtain a good separation of the different sized beads [Figs. 10(d)-10(f)]. The data points from the experiments in Figs. 10(d)-10(f) are added as circles in (b) of Fig. 7. Although, the settings in Fig. 10(d) are slightly below the theoretical critical settings, yet a spillover of the particles is observed, likely a result of the experimental sensitivity towards close-to-critical settings. As the acoustic field in the main channel moves the particles towards the central pressure node in addition to the inertia-induced lateral shift, it is advised to choose flow settings carefully in regards to the critical settings in order to guarantee an efficient particle separation.

\section{CONCLUSION}

High sample throughput larger than $100 \mu 1 \mathrm{~min}^{-1}$ is key for a clinically relevant enrichment of rare cells, such as scarce circulating tumor cells in a liquid biopsy sample. However, at elevated flow rates in a standard CTC acoustophoresis separation channel, we observe an inertiainduced phenomenon, i.e., the increased deformation of the flow separatrices, that may compromise separation performance. The phenomena is studied experimentally and theoretically at increased flow rates, revealing a lateral relocation of the particle trajectories, flow separatrices and stagnation streamlines at the inlet and outlet flow splitters. At specific flow settings the inertia-induced effect caused all particles, independent of size, to exit via the center outlet, leading to a breakdown of the particle separation. These findings will assist in chip optimization for maximized sample throughput, and we believe that additional improvements of the sample processing will be achieved through such acoustofluidic design advancements.

\section{ACKNOWLEDGMENTS}

This paper is supported by the Swedish Research Council (Grants No. 2019-00795 and No. 2018-03672), and the Swedish Foundation for Strategic Research (Grant No. SBE13-0049).

W.Q. is supported by MSCA EF Seal of Excellence IF2018 from Vinnova, Sweden's Innovation Agency (Grant No. 2019-04856).

The spinning disk confocal microscope used in this study is acquired by the funding from the European Research Council (ERC) under the European Union's Horizon 2020 research and innovation programme (Grant Agreement No. 852590).

\section{APPENDIX}

\section{Estimation of the lift force in the main channel}

In microfluidic channels, inertia can lead to a migration of the particles towards an equilibrium position [21]. The particle Reynolds number is given by [24]

$$
R_{p}=\frac{\rho u_{\max } \ell_{p}}{\eta}\left(\frac{\ell_{p}}{\ell_{c}}\right)
$$

where $\ell_{p}$ is the characteristic size of the particle, which, for spherical particles, equals the particle radius $r_{p}$, and $\ell_{c}$ is the characteristic length of the channel, which, for a rectangular channel, can be approximated by the hydraulic channel radius $r_{h}$ [25]. For the present case we have

$$
r_{h}=\frac{H \times W}{H+W}=107.143 \mu \mathrm{m},
$$

where we use the height $H$ and width $W$ of the channel. Finally, $u_{\max }$ is the maximum fluid velocity inside the channel without the particle. For our aspect ratio and a total flow rate $Q$ the maximal fluid velocity can be evaluated as [26]

$$
u_{\max }=1.92 \frac{Q}{H W} .
$$

Migration effects are apparent when $R_{p} \approx 1$ or greater $[21,25]$. By considering a flow rate of $800 \mu \mathrm{l} \mathrm{min}^{-1}$ in the main channel and a particle radius ranging from 1 to $5 \mu \mathrm{m}$, we obtain a Reynolds number in the order of $10^{-3}$ to $10^{-1}$, due to the very small $r_{p} / r_{h}$ ratio in our system.

However, due to the length of the separation channel, i.e., $L \simeq 40 \mathrm{~mm}$, we cannot conclude a priori that a weak lift force produces an insignificant displacement of the particle between the inlet and the outlet sections of the separation channel.

The order of magnitude of this displacement $\Delta x=x_{\text {in }}-$ $x_{\text {out }}$ can be estimated by considering the ideal case of a particle moving under the action of a constant lift force 


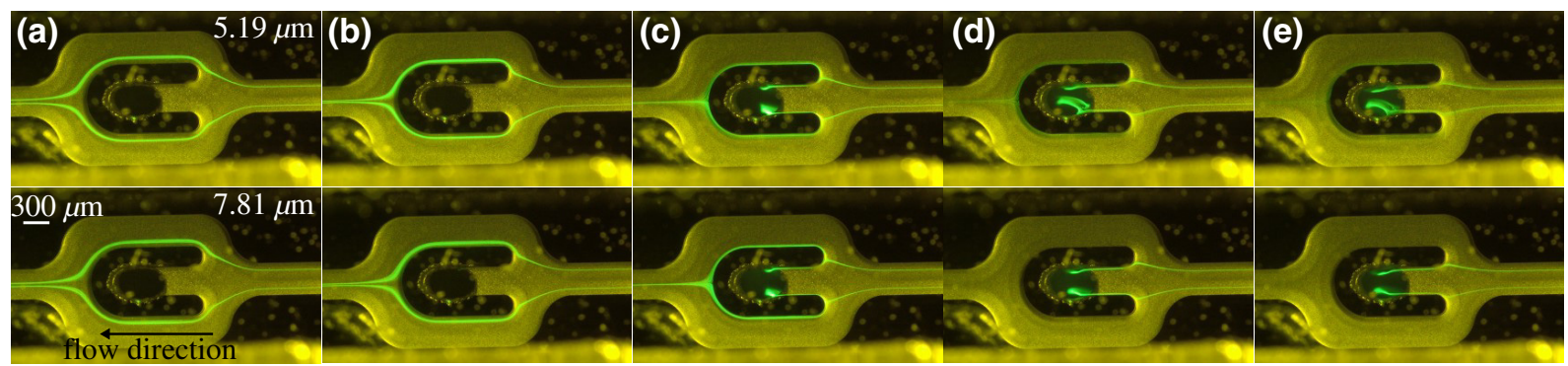

FIG. 12. For a given splitting ratio, spillover occurred at a specific flow rate independent of particle size. The images show $5.19 \mu \mathrm{m}$ (top row) and $7.81 \mu \mathrm{m}$ (bottom row) polystyrene particles at $r_{\text {in }}=0.65$ and $r_{\text {out }}=0.35$ at a total flow rate of (a) $200 \mu 1 \mathrm{~min}^{-1}$,

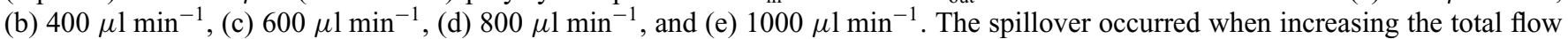
rate from $400 \mu 1 \mathrm{~min}^{-1}$ to $600 \mu 1 \mathrm{~min}^{-1}$ for both particle sizes.

$F_{l}$ along the entire separation channel. In this case the displacement can be written as

$$
\Delta x=\frac{U_{x}}{U_{y}} L .
$$

For a very small $r_{p} / r_{h}$ ratio and far from the walls, the lift force is approximately given by

$$
F_{l} \approx \eta u_{\max } R_{p}\left(\frac{r_{p}}{r_{h}}\right)^{2} .
$$

Balance the lift force and the Stokes drag then yields the estimate for the lift velocity

$$
U_{x} \approx \frac{F_{l}}{6 \pi \eta r_{p}}=\frac{u_{\max } R_{p} r_{p}}{6 \pi r_{h}^{2}} .
$$

Since the average axial velocity is given by

$$
U_{y}=\frac{Q}{H W},
$$

the overall lift velocity ratio is estimated as

$$
\frac{U_{x}}{U_{y}} \approx \frac{F_{l}}{6 \pi \eta r_{p} U_{y}}=\frac{1.92 R_{p} r_{p}}{6 \pi r_{h}^{2}} \sim 10^{-6} .
$$

The total lateral displacement due to the lift force is then approximately

$$
\Delta x \approx 10^{-3} r_{p} .
$$

Therefore, we conclude that in our experiments the lift force does not contribute to an additional lateral displacement of the particles towards the center of the separation channel.

\section{Size dependency of induced spillover effect}

Fluorescent $7.81 \mu \mathrm{m}$ (FluoGreen, microparticles $\mathrm{GmbH}$ ) polystyrene particles are suspended in FACS buffer and run through the system. Previously used $5.19-\mu \mathrm{m}$ beads are run as reference. Four flow splitting ratios (using $r_{\text {in }}+$ $r_{\text {out }}=1$ the inlet splitting ratios $r_{\text {in }}$ are $0.20,0.40,0.65$, and 0.75$)$ and five total flow rates $(200,400,600,800$, and $1000 \mu 1 \mathrm{~min}^{-1}$ ) are employed. Images are collected for visual analysis.

To study if the inertia-induced effect is size dependent, two different-sized particles are employed. The particle trajectories for the $5.19-\mu \mathrm{m}$ and the $7.81-\mu \mathrm{m}$ beads showed no difference in lateral displacement at the outlet fork for any investigated splitting ratio and flow rate. For
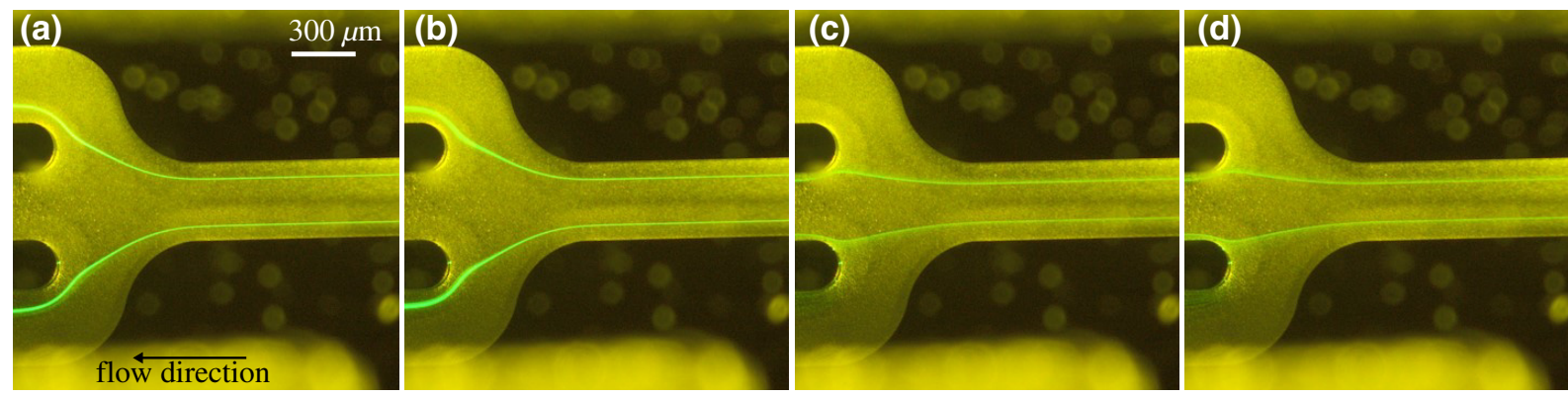

FIG. 13. The lateral displacement of the particle trajectories at increased flow rates are not due to an acoustic effect from the prefocusing PZT. Particle trajectories $\left(5.19 \mu \mathrm{m}\right.$ ) at the outlet fork at (a) $200 \mu 1 \mathrm{~min}^{-1}$ and $8 \mathrm{~V}_{\mathrm{pp}}$ (b) $200 \mu 1 \mathrm{~min}^{-1}$ and $17 \mathrm{~V}_{\mathrm{pp}}$ (c) $1000 \mu 1 \mathrm{~min}^{-1}$ and $17 \mathrm{~V}_{\mathrm{pp}}$ and (d) $1000 \mu 1 \mathrm{~min}^{-1}$ and $12 \mathrm{~V}_{\mathrm{pp}}$ are shown. The major shift occurs when increasing the flow rate to $1000 \mu \mathrm{min}^{-1}$, and it is not reduced by a lowering of the prefocusing voltage. 
$r_{\text {in }}=0.65$ with $r_{\text {out }}=0.35$ flow splitting ratios (Fig. 12) and $r_{\text {in }}=0.75$ with $r_{\text {out }}=0.25$ ratios, the spillover of particles into the center outlet occurred at the same flow rate for the two particle sizes. The results demonstrate that the inertia-induced hydrodynamic effect is independent of particle size.

\section{Prefocusing influence on induced spillover effect}

Fluorescent $5.19-\mu \mathrm{m}$ polystyrene particles (FluoGreen, microparticles $\mathrm{GmbH}$ ) are suspended in FACS buffer and a fixed splitting ratio of $r_{\text {in }}=0.65$ and $r_{\text {out }}=0.35$ is used. The piezoelectric transducer is actuated at $4.8 \mathrm{MHz}$ and $8 \mathrm{~V}_{\mathrm{pp}}$, and the flow rate is set to $200 \mu \mathrm{l} \mathrm{min}^{-1}$. The voltage is then increased to the maximum output of $17 \mathrm{~V}_{\mathrm{pp}}$, and thereafter kept constant while increasing the flow rate to $1000 \mu 1 \mathrm{~min}^{-1}$. Finally, the voltage is reduced to $12 \mathrm{~V}_{\mathrm{pp}}$ while keeping the flow rate constant at $1000 \mu 1 \mathrm{~min}^{-1}$. Images over the outlet fork are collected for the different flow rates and voltages and the particle trajectories for the different settings are compared.

To investigate any influence of the acoustic prefocusing on the lateral shift of the particles, the prefocusing voltage is tuned while tracking the particle trajectories. When increasing the prefocusing actuation voltage from $8 \mathrm{~V}_{\mathrm{pp}}$ to $17 \mathrm{~V}_{\mathrm{pp}}$ while running at $200 \mu 1 \mathrm{~min}^{-1}$, a small shift in the lateral position of the particles is observed [Figs. 13(a) and 13(b)]. Due to the low flow rate, the particles flowing in the channel have a long retention time and any influence from the prefocusing actuation in the main channel has a possibility to affect the suspended particles. This small shift is in the range of $10 \%-15 \%$ of the lateral displacement seen when running at maximal throughput. After increasing the flow rate to $1000 \mu \mathrm{min}^{-1}$ and keeping the voltage fixed at $17 \mathrm{~V}_{\mathrm{pp}}$, the larger shift in the lateral position of the particles can be seen, despite the now much shorter retention time [Fig. 13(c)]. This shows that the lateral displacement occurring when increasing the flow rate is not due to an acoustic effect from the prefocusing. Lowering the voltage to $12 \mathrm{~V}_{\mathrm{pp}}$ while keeping the flow rate constant at $1000 \mu 1 \mathrm{~min}^{-1}$ did not alter the position of the particles [Fig. 13(d)]. Again, proving that the shift is not a result of any acoustic effects.

\section{Derivation of the centrifugal drift}

While the particles follow the motion of the fluid around corners, they will be subjected to centrifugal acceleration. The question arises to what extent the centrifugal acceleration will push particles across streamlines. We analytically show that the centrifugal drift of the particles here is in the nanometre range, and therefore it can be assumed that the path of a particle is overlapping the corresponding streamline.

As shown in Fig. 14, the particle coordinates are given by the $\mathbf{r}(t)$ vector with the components $x(t)$ and $y(t)$ given

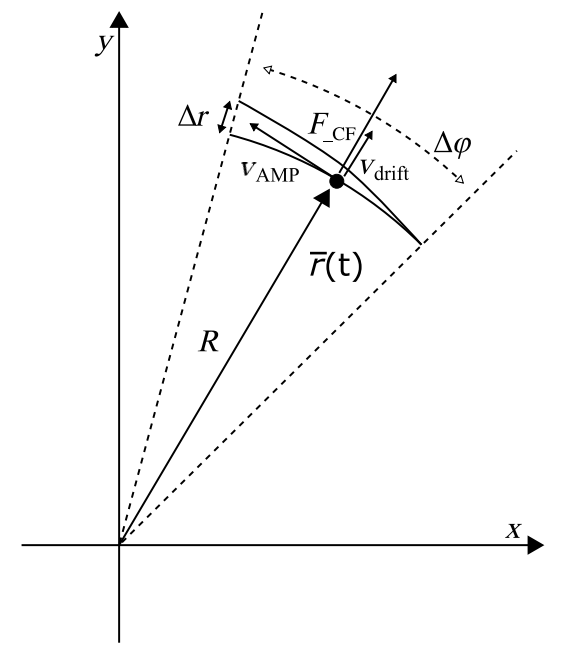

FIG. 14. A particle is moving on a circular path of radius $R$ and currently at position $\bar{r}(t)$. The velocity along the circular path is $v_{\text {AMP. }}$. Due to the particle inertia, the particle experiences the centrifugal acceleration, modeled as centrifugal drift force $F_{\mathrm{CF}}$. The centrifugal force leads to a drift velocity that deviates the particle from its circular path when it is not counteracted. Here, the particle is surrounded by a fluid, and we assume Stokes drag to model the drift velocity. As the particle experiences a drift velocity along a path length of $R \times \Delta \varphi$ it will be moved outwards by $\Delta r$.

by

$$
\begin{aligned}
& x(t)=R \cos (\omega t), \\
& y(t)=R \sin (\omega t),
\end{aligned}
$$

the acceleration of the particle is then

$$
\begin{aligned}
& \ddot{x}(t)=-R \omega^{2} \cos (\omega t), \\
& \ddot{y}(t)=-R \omega^{2} \sin (\omega t),
\end{aligned}
$$

and the velocity amplitude along the circular path is $v_{a}=R \omega$.

The centrifugal acceleration can be interpreted as a centrifugal force

$$
\left|F_{\mathrm{CF}}\right|=\frac{v_{a}^{2}}{R} m_{\mathrm{eff}}
$$

where we introduce the effective mass $m_{\mathrm{eff}}=(4 / 3) a^{3}$ $\left(\rho_{p}-\rho_{f}\right)$. Balancing the Stokes drag with the centrifugal drift force, we arrive at the drift velocity amplitude $v_{\text {drift }}$

$$
v_{\text {drift }}=F_{\mathrm{CF}} / s_{D} \Rightarrow v_{\text {drift }}=\frac{v_{a}^{2} m_{\mathrm{eff}}}{s_{D}} .
$$

Multiplying the drift velocity $v_{\text {drift }}$ with the total time the particle spends in the arc we arrive at the total drift 
TABLE II. Physical parameters used for assessing the centrifugal drift.

\begin{tabular}{lc}
\hline \hline Parameter & Value \\
\hline Height & $h=150 \mu \mathrm{m}$ \\
Width & $w=375 \mu \mathrm{m}$ \\
Radius of curvature & $R=100 \mu \mathrm{m}$ \\
Particle diameter & $d=2 \times a=5 \mu \mathrm{m}$ \\
Particle density & $\rho_{p}=1050 \mathrm{~kg} \mathrm{~m}^{-3}$ \\
Water dynamic viscosity & $\eta=0.001 \mathrm{~Pa} \mathrm{~s}$ \\
Stokes drag coefficient & $s_{D}=6 \pi \mathrm{a \eta}$ \\
Traveled angle & $\Delta \varphi$ \\
Traveled distance along the arc & $\Delta \varphi \times R$ \\
Travel time $\Delta t$ & $\Delta \varphi \times R / v_{a}$ \\
Drift distance & $\Delta r=\Delta \varphi m_{\mathrm{eff}} v_{a} / s_{D}$ \\
Effective mass & $m_{\mathrm{eff}}=4 / 3 \varphi a^{3}\left(\rho_{p}-\rho_{f}\right)$ \\
Velocity along the arc & $v_{a}$ \\
\hline \hline
\end{tabular}

displacement $\Delta r$, formally,

$$
\Delta r=\Delta t \times v_{\mathrm{drift}}=\frac{\Delta \varphi v_{a} m_{\mathrm{eff}}}{s_{D}} .
$$

Then, using the values from Table II we find $\Delta \varphi=$ $\pi / 2, v_{a}=0.3 \mathrm{~m} \mathrm{~s}^{-1}, m_{\mathrm{eff}}=3.3 \times 10^{-15} \mathrm{~kg}$, and $s_{D}=$ $4.7 \times 10^{-8} \mathrm{~N} \mathrm{~s} \mathrm{~m}^{-1}$ yields a drift distance of $33 \mathrm{~nm}$. Thus, the centrifugal drift is very small and the particle paths can be assumed to be coincident with the corresponding streamlines.

\section{Mesh study}

A mesh study is performed to find the optimal trade-off between performance and accuracy. Six predefined meshes from COMSOL Multiphysics ranging from coarser to extra fine are included in the mesh study, Fig. 15. We choose

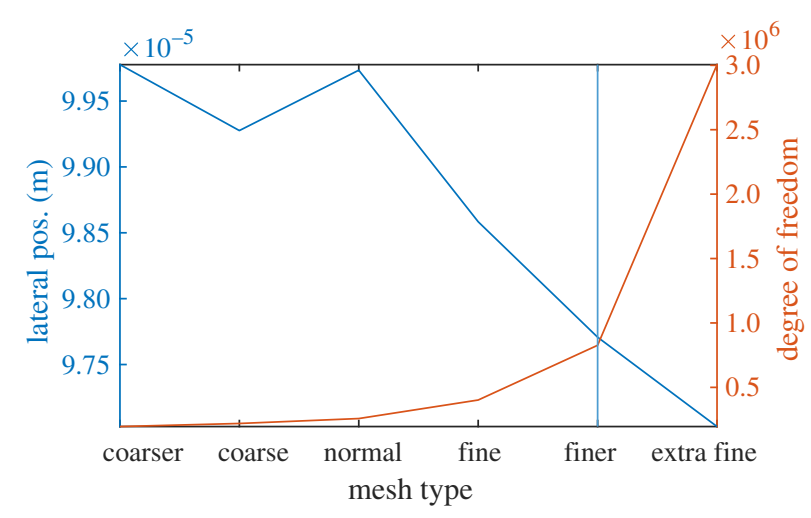

FIG. 15. The mesh is selected to achieve the best trade-off between computational time and accuracy. The selected mesh is indicated by the vertical line and offers a $1-\mu \mathrm{m}$ precision with respect to the most precise mesh that we could use. As a mesh criterion, we choose the lateral $(x)$ position of the particle streamline computed for a total flow rate of $800 \mu 1 \mathrm{~min}^{-1}$ close to the outlet of the inlet fork. the finer mesh that gives a 1- $\mu$ m accuracy in the lateral $(x)$ position of the particle streamline at the outlet of the inlet fork.

[1] F. Petersson, A. Nilsson, C. Holm, H. Jönsson, and T. Laurell, Separation of lipids from blood utilizing ultrasonic standing waves in microfluidic channels, Analyst 129, 938 (2004).

[2] C. Grenvall, P. Augustsson, J. R. Folkenberg, and T. Laurell, Harmonic microchip acoustophoresis: A route to online raw milk sample precondition in protein and lipid content quality control, Anal. Chem. 81, 6195 (2009).

[3] C. Magnusson, P. Augustsson, A. Lenshof, Y. Ceder, T. Laurell, and H. Lilja, Clinical-scale cell-surface-marker independent acoustic microfluidic enrichment of tumor cells from blood, Anal. Chem. 89, 11954 (2017).

[4] K. Cushing, E. Undvall, Y. Ceder, H. Lilja, and T. Laurell, Reducing wbc background in cancer cell separation products by negative acoustic contrast particle immunoacoustophoresis, Anal. Chim. Acta. 1000, 256 (2018).

[5] A. Urbansky, F. Olm, S. Scheding, T. Laurell, and A. Lenshof, Label-free separation of leukocyte subpopulations using high throughput multiplex acoustophoresis, Lab on a Chip 19, 1406 (2019).

[6] E. Ozkumur, A. M. Shah, J. C. Ciciliano, B. L. Emmink, D. T. Miyamoto, E. Brachtel, M. Yu, P.-i. Chen, B. Morgan, and J. Trautwein, et al., Inertial focusing for tumor antigen-dependent and-independent sorting of rare circulating tumor cells, Sci. Trans. Med. 5, 179ra47 (2013).

[7] N. Nivedita and I. Papautsky, Continuous separation of blood cells in spiral microfluidic devices, Biomicrofluidics 7, 054101 (2013).

[8] J. Zhang, D. Yuan, R. Sluyter, S. Yan, Q. Zhao, H. Xia, S. H. Tan, N.-T. Nguyen, and W. Li, High-throughput separation of white blood cells from whole blood using inertial microfluidics, IEEE Trans. Biomed. Circuits Syst. 11, 1422 (2017).

[9] H. Ramachandraiah, H. A. Svahn, and A. Russom, Inertial microfluidics combined with selective cell lysis for high throughput separation of nucleated cells from whole blood, Rsc Adv. 7, 29505 (2017).

[10] S. C. Hur, A. J. Mach, and D. Di Carlo, High-throughput size-based rare cell enrichment using microscale vortices, Biomicrofluidics 5, 022206 (2011).

[11] A. J. Mach, J. H. Kim, A. Arshi, S. C. Hur, and D. Di Carlo, Automated cellular sample preparation using a centrifugeon-a-chip, Lab on a Chip 11, 2827 (2011).

[12] E. Sollier, D. E. Go, J. Che, D. R. Gossett, S. O’Byrne, W. M. Weaver, N. Kummer, M. Rettig, J. Goldman, and N. Nickols, et al., Size-selective collection of circulating tumor cells using vortex technology, Lab on a Chip 14, 63 (2014).

[13] J. A. Davis, D. W. Inglis, K. J. Morton, D. A. Lawrence, L. R. Huang, S. Y. Chou, J. C. Sturm, and R. H. Austin, Deterministic hydrodynamics: Taking blood apart, Proc. National Acad. Sci. 103, 14779 (2006).

[14] M. Yamada and M. Seki, Hydrodynamic filtration for on-chip particle concentration and classification utilizing microfluidics, Lab on a Chip 5, 1233 (2005). 
[15] A. Urbansky, P. Ohlsson, A. Lenshof, F. Garofalo, S. Scheding, and T. Laurell, Rapid and effective enrichment of mononuclear cells from blood using acoustophoresis, Sci. Rep. 7, 1 (2017).

[16] H. Jönsson, C. Holm, A. Nilsson, F. Petersson, P. Johnsson, and T. Laurell, Particle separation using ultrasound can radically reduce embolic load to brain after cardiac surgery, Ann. Thoracic Surg. 78, 1572 (2004).

[17] P. K. Kundu, I. M. Cohen, and D. Dowling, Fluid mechanics 5 th (2012).

[18] E. M. L. L. D. Landau, Lehrbuch der theoretischen physik, vi hydrodynamik (2014).

[19] B. Chun and A. Ladd, Inertial migration of neutrally buoyant particles in a square duct: An investigation of multiple equilibrium positions, Phys. Fluids 18, 031704 (2006).

[20] E. S. Asmolov, The inertial lift on a spherical particle in a plane poiseuille flow at large channel reynolds number, J. Fluid Mech. 381, 63 (1999).

[21] D. Di Carlo, Inertial microfluidics, Lab on a Chip 9, 3038 (2009).
[22] H. Amini, E. Sollier, M. Masaeli, Y. Xie, B. Ganapathysubramanian, H. A. Stone, and D. Di Carlo, Engineering fluid flow using sequenced microstructures, Nat. Commun. 4, 1 (2013).

[23] A. Urbansky, A. Lenshof, J. Dykes, T. Laurell, and S. Scheding, Affinity-bead-mediated enrichment of cd8+ lymphocytes from peripheral blood progenitor cell products using acoustophoresis, Micromachines 7, 101 (2016).

[24] B. Ho and L. Leal, Inertial migration of rigid spheres in two-dimensional unidirectional flows, J. Fluid Mech. 65, 365 (1974).

[25] D. Di Carlo, D. Irimia, R. G. Tompkins, and M. Toner, Continuous inertial focusing, ordering, and separation of particles in microchannels, Proc. National Acad. Sci. 104, 18892 (2007).

[26] F. Delplace, Laminar flow of newtonian liquids in ducts of rectangular cross-section a model for both physics and mathematics, Open Access J. Math. Theor. Phys. 1, 198 (2018). 